\title{
Stretching weakly bending filaments with spontaneous curvature in two dimensions
}

\author{
Panayotis Benetatos and Eugene M. Terentjev \\ Cavendish Laboratory, University of Cambridge, J. J. Thomson Avenue, Cambridge, CB3 OHE, United Kingdom
}

(Dated: October 26, 2018)

\begin{abstract}
Some important biomolecules (for instance, bacterial FtsZ and eukaryotic DNA) are known to posses spontaneous (intrinsic) curvature. Using a simple extension of the wormlike chain model, we study the response of a weakly bending filament in two dimensions to a pulling force applied at its ends (a configuration common in classical in-vitro experiments and relevant to several in-vivo cell cases). The spontaneous curvature of such a chain or filament can in general be arc-length dependent and we study a case of sinusoidal variation, from which an arbitrary case can be reconstructed via Fourier transformation. We obtain analytic results for the force-extension relationship and the width of transverse fluctuations. We show that spontaneous-curvature undulations can affect the forceextension behavior even in relatively flexible filaments with a persistence length smaller than the contour length.
\end{abstract}

PACS numbers: 36.20.Ey,87.15.ad,82.37.Rs

\section{INTRODUCTION}

Semiflexible polymers are fluctuating macromolecules with a bending stiffness intermediate between that of a random coil (Gaussian chain) and a rigid rod. They have been the subject of intense experimental and theoretical study in recent years because many important biomolecules fall in this category. The elastic behavior of the structural elements of the cytoskeleton (F-actin, microtubules, intermediate filaments) as well as that of DNA strongly depend on their bending stiffness [1, 2].

A widely used minimal model of semiflexible polymers is the wormlike chain model (WLC) which treats the filament as a locally inextensible one-dimensional fluctuating curve with bending stiffness [3, 4. This model involves only two length scales, namely, the contour length $L$ and the persistence length $L_{p}$, which is the correlation length of the polymer directedness related to the chain bending modulus $\kappa=\frac{1}{2} L_{p} k_{B} T(d-1)$, where $d$ is the dimensionality of the embedding space. In the limit $L / L_{p} \gg 1$ the semiflexible chain transforms into a Gaussian coil and the question of excluded-volume interaction may become relevant; in the opposite limit $L / L_{p} \ll 1$ the chain approaches the limit of rigid rod, in which case the additional modulus penalizing the local stretching/compression may come into play [5]. Despite its simplicity, the WLC model has been successfully used to describe some important experimental results such as the strong stretching of ds-DNA [6]. However, the advent of single molecule experiments has revealed many occasions where the complexity of the microscopic architecture of the filament appears to affect its macroscopic behavior in a way that cannot be accounted for by the classical WLC model.

An important property of some biomolecules which goes beyond the basic WLC model is spontaneous local curvature. FtsZ is a protein of the prokaryotic cytoskeleton which plays an essential role in cell division as it assembles into the contractile ring (Z-ring) that divides the bacterium [7]. It can be visualized on a mica surface using atomic-force microscopy (AFM). An important property of many FtsZ protofilaments is the spontaneous curvature of their relaxed state. Their role in force generation strongly depends on the determination of their persistence length 8. Assuming the relaxed state of FtsZ protofilaments to be straight, Dajkovic et al. 9 estimated $L_{p} \approx 200 \mathrm{~nm}$ which is much too small. On the other hand, Horger et al. [10] taking into account the finite spontaneous curvature measured by AFM (average radius of curvature $\approx 100 \mathrm{~nm}$ ), determined a persistence length $L_{p} \approx 4 \mu \mathrm{m}$ which is close to the value calculated from models of the flexural rigidity.

The existence of stable bent configurations of DNA has been known for many years and they play important roles in many basic genetic processes [11. Spontaneous curvature in the local structure of DNA is sequence-dependent 12. As in the case of FtsZ, the presence (or absence) of local spontaneous curvature in a DNA filament is crucial in determining the persistence length and its relation to the bending rigidity. In a recent experiment, AFM imaging of two-dimensional human DNA chains and comparison to measurements on both intrinsically straight and uncorrelated HCV DNA has demonstrated their spontaneous curvature [13].

The theoretical study of small (Gaussian) thermal fluctuations of three-dimensional filaments with spontaneous curvature and torsion has yielded analytical expressions for the orientational correlation functions and the persistence length [14. An auxiliary field theory of threedimensional polymers with intrinsic curvature has obtained the orientational correlations, mean square separation and the force-extension relation of such chains in the $L / L_{p} \gg 1$ limit, showing that helical configurations arise due to the excluded-volume interaction [15. Real filaments with spontaneous curvature in three dimensions also possess a finite twist rigidity which has been shown 
to affect the end-to-end distribution [16] and the response to a stretching force giving rise to instabilities [17, 18]. The zero-temperature conformations of two-dimensional filaments with constant spontaneous curvature under an applied force for various boundary conditions, as well as the linear response at finite temperature to an applied force, have been recently investigated in 19 .

In this paper, we study the response of a twodimensional WLC with spontaneous curvature to a stretching force applied at the ends. Although in general one of course expects the stretched chain to explore fluctuations in both perpendicular directions, the twodimensional limit is a meaningful simplifying approximation that permits us to carry out a complete analytical study, while retaining some of the relevant qualitative features of the general problem. The three-dimensional problem is more complicated in two nontrivial aspects. Curvature in $3 d$ is always positive definite whereas in $2 d$ we can define the signed curvature. In addition, the presence of twist rigidity complicates matters even further [16 18]. As we explain in Sec. VII, however, the main conclusion of this work which is the softening of the stretching response due to a spatially varying spontaneous curvature is expected to carry through in $3 d$. Besides, many imaging experimental investigations [10, 13 . are carried out on molecules confined to a surface. A stretching experiment on DNA electrostatically confined to the surface of a fluid membrane is presented in [20. The elasticity of semiflexible polymers without spontaneous curvature in two dimensions has been studied analytically in [21. The spontaneous curvature can in general be inhomogeneous, dependent on the location along the arc length $s$ along the chain. Any such variation can be decomposed into Fourier harmonics and so we study a case of local curvature varying in an arbitrary sinusoidal fashion along the chain length. We assume that the filament is weakly bending. This approximation can be realized in the case of filaments with small spontaneous curvature and small thermal fluctuations or in the case of strong stretching forces which have flattened out the initially large spontaneous curvature and/or the large thermal undulations. This is the simplest possible extension of the WLC model to include spontaneous curvature analytically. A formally similar approach has recently been employed in 22] to study uncorrelated quenched disorder in the spontaneous curvature of stretched twodimensional filaments.

The paper is organized as follows. In Sec. II we introduce our model and derive the Gaussian energy functional which characterizes the stretched polymer. In the absence of thermal fluctuations, the polymer assumes the ground-state conformation which minimizes the elastic energy for given boundary conditions; finding this conformation is the subject of Sec. III. In Sec. IV we show that thermal fluctuations decouple from the athermal mechanical response and are independent of the spontaneous

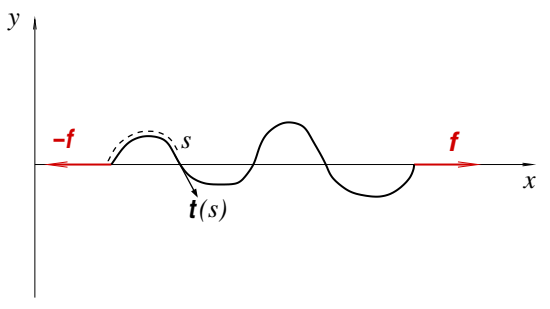

FIG. 1: Schematic diagram of a filament with spatially varying spontaneous curvature showing the notation used.

curvature. In Sec. V we calculate the force-extension relationship and identify the various force scales in the problem, while in Sec. VI we investigate the response of the shape of transverse fluctuations on the stretching force. We conclude and discuss a possible extension of this work in Sec. VII.

\section{MODEL}

We treat a semiflexible polymer with spontaneous (intrinsic) curvature by simply extending the classical WLC model. The filament is described as a plane curve, $\mathbf{r}(s)$, where $s \in[0, L]$ is the arc-length along its contour, subject to a local inextensibility constraint so that the total contour length $L$ is constant. The constraint is mathematically expressed by the condition:

$$
\left|\frac{\partial \mathbf{r}(s)}{\partial s}\right|=1 \text {. }
$$

The effective free-energy functional ("Hamiltonian") of the polymer depends only on its curvature (bending),

$$
\mathcal{H}_{0}[\mathbf{r}(s)]=\frac{\kappa}{2} \int_{0}^{L} d s\left[\left|\frac{\partial \mathbf{t}(s)}{\partial s}\right|-c(s)\right]^{2},
$$

where $\mathbf{t}(s)=\partial \mathbf{r}(s) / \partial s$ is the tangent vector and $c(s)$ is the spontaneous curvature at the position $s$, cf. Fig.1. This bending energy penalizes conformations with a local curvature if it deviates away from $c(s)$.

The interaction with a stretching force $\mathbf{f}$ which pulls at the polymer end-points is expressed by adding to $\mathcal{H}_{0}$ the work function

$$
\mathcal{W}[\mathbf{r}(s)]=-\mathbf{f} \cdot[\mathbf{r}(L)-\mathbf{r}(0)]=-\int_{0}^{L} d s \mathbf{f} \cdot \mathbf{t}(s) .
$$

The stretching response of a filament described by Eqs. $(2)$ and (3) is in general analytically intractable for two reasons. One is the well-known problem associated with the local inextensibility constraint, the other is the nonanalyticity introduced by the definition of curvature as the modulus of $d \mathbf{t}(s) / d s$. However, in the present case of weakly-bending filaments in two dimensions the problem simplifies drastically and becomes linear. The coordinate system that we use is shown in Fig. 1. To describe 
a weakly-bending filament, it is convenient to use the parametrization $\mathbf{r}(s)=[s-X(s), y(s)]$ which implies $\mathbf{t}(s)=\left[1-\partial_{s} X, t_{\perp}(s)\right]$. The inextensibility condition can be written as $t_{\perp}^{2}+\left(1-\partial_{s} X\right)^{2}=1$ which, in the weakly bending approximation $\left|t_{\perp}(s)\right| \ll 1$, yields $\partial_{s} X=\frac{1}{2} t_{\perp}^{2}+$ $\mathcal{O}\left(t_{\perp}^{4}\right)$ and therefore $\partial_{s}^{2} X=t_{\perp} \partial_{s} t_{\perp}+\frac{1}{2} t_{\perp}^{3} \partial_{s} t_{\perp}+\mathcal{O}\left(\overline{t_{\perp}^{5}}\right)$. In this approximation, the principal normal to the filament can be considered perpendicular to its backbone (x-direction) and $\partial \mathbf{t} / \partial s \approx\left[0, \partial_{s} t_{\perp}\right]$. Introducing the signed curvature, $\tilde{c}(s)$, we can express the energy of a weakly-bending stretched filament as

$$
\begin{aligned}
\mathcal{H}[y(s)]= & \frac{\kappa}{2} \int_{0}^{L} d s\left[\left(\frac{\partial^{2} y(s)}{\partial s^{2}}\right)-\tilde{c}(s)\right]^{2} \\
& +\frac{1}{2} f \int_{0}^{L} d s\left(\frac{\partial y(s)}{\partial s}\right)^{2}-f L .
\end{aligned}
$$

$\tilde{c}(s)$ is positive if the filament bends convexly and negative if it bends concavely.

\section{GROUND STATE}

In the absence of thermal fluctuations, the response of the filament to the stretching force is determined by minimizing the functional $\mathcal{H}[y(s)]$ for appropriate boundary conditions. In this paper, we assume hinged-hinged boundary conditions which can be realized by attaching beads at the end-points that can exert forces but not moments [26]. As we show below, in the case of strong stretching, the specific boundary conditions may become irrelevant. Formally, they are expressed by the following relations:

$$
\begin{aligned}
& y(0)=y(L)=0 \\
& y^{\prime \prime}(0)=\tilde{c}(0), y^{\prime \prime}(L)=\tilde{c}(L) .
\end{aligned}
$$

(The primes denote derivatives with respect to s.) The minimizing configuration, $y_{a}(s)$, obeys an EulerLagrange equation which can be obtained by adding an infinitesimal variation, $y(s)=y_{a}(s)+\epsilon \eta(s)$, where $\epsilon$ is infinitesimal and $\eta(s)$ is an arbitrary function of $s$ satisfying $\eta(0)=\eta(L)=0$ and $\eta^{\prime \prime}(0)=\eta^{\prime \prime}(L)=0$ and requiring the derivative of $\mathcal{H}(\epsilon)$ as a function of $\epsilon$ to vanish. The athermal configuration is the solution to the equation

$$
-f y_{a}^{\prime \prime}(s)+\kappa y_{a}^{\prime \prime \prime \prime}(s)-\kappa \tilde{c}^{\prime \prime}(s)=0
$$

with the boundary conditions given by Eq. (5).

We first consider the case of constant curvature: the value $\tilde{c}(s)=c_{0}$ is maintained along the chain. The last term in Eq. (6) vanishes and the athermal configuration $y_{a}(s)$ is determined by hyperbolic functions with the characteristic length scale $l_{f} \equiv \sqrt{\kappa / f}$. Implementing the boundary conditions, we obtain

$$
\begin{aligned}
& y_{a}(s)=\frac{c_{0} \kappa}{f \sinh \left(\frac{L}{l_{f}}\right)}\left[\sinh \left(\frac{s}{l_{f}}\right)+\sinh \left(\frac{(-s+L)}{l_{f}}\right)\right. \\
& \left.-\sinh \left(\frac{L}{l_{f}}\right)\right] .
\end{aligned}
$$

As expected, in the vanishing-force limit $f \rightarrow 0$ the above expression yields a parabola, $y_{a}(s)=\frac{1}{2} c_{0} s(s-L)$, which is identical, to leading order in the weakly bending approximation, to a circular arc of curvature $c_{0}$. For $l_{f} \ll L$, and $l_{f} \ll s \ll L-l_{f}$, we get a uniform displacement, $y_{a}(s)=-c_{0} \kappa / f$, which implies that the actual response comes from the segments of arc-length $l_{f}$ at the boundaries.

For a filament with arbitrary variable spontaneous curvature, one can expand it in Fourier modes:

$$
\tilde{c}(s)=\sum_{n} c_{n} \cos \left(q s+\phi_{n}\right), q \equiv \frac{n \pi}{L}, n \in \mathbb{N}
$$

Since Eq. (6) is linear, its solution for the general case will be the superposition of the modes which correspond to the individual harmonics. Taking a particular mode, $\tilde{c}(s)=c_{n} \cos (q s+\phi)$, the ground-state stretched configuration becomes:

$$
y_{a}(s)=\frac{\kappa c_{n}}{\left(f+q^{2} \kappa\right) \sinh \left(\frac{L}{l_{f}}\right)}\left[2 \cos (\phi) \sinh \left(\frac{1}{2} \frac{L}{l_{f}}\right) \cosh \left(\frac{1}{2} \frac{(L-2 s)}{l_{f}}\right)-\cos (q s+\phi) \sinh \left(\frac{L}{l_{f}}\right)\right] .
$$

Since Eq. (6) is linear, a chain with spontaneous curvature containing many harmonic modes yields a response $\left(y_{a}(s)\right)$ which is the superposition of the responses for the individual modes. The above expression becomes more transparent in the case where $\tilde{c}(s)=c_{0} \sin (q s)$,

$$
y_{a}(s)=-\frac{c_{0} \kappa \sin (q s)}{f+q^{2} \kappa}
$$

In the strong stretching regime, $l_{f} \ll L$, any mode yields 
a uniform response for $l_{f} \ll s \ll L-l_{f}$,

$$
y_{a}(s)=-\frac{c_{n} \kappa \cos \left(q s+\phi_{n}\right)}{f+q^{2} \kappa},
$$

consisting of a simple reduction in the amplitude. We see that the length scale $l_{f}$ defines the distance along the chain over which the boundary effects persist (boundary layer).

\section{RESPONSE AT FINITE TEMPERATURE}

Thermal fluctuations are added to the athermal ground-state response, $y(s)=y_{a}(s)+u(s)$, and contribute according to a Boltzmann weight associated with the energy given in Eq. (4). As we show below, in the linear theory of the weakly-bending approximation, the two Gaussian contributions (thermal and athermal) decouple. The elastic energy of thermal fluctuations then takes the form,

$$
\begin{aligned}
& \mathcal{H}[u(s)]=\frac{\kappa}{2} \int_{0}^{L} d s\left(u^{\prime \prime}(s)\right)^{2}+\frac{f}{2} \int_{0}^{L} d s\left(u^{\prime}(s)\right)^{2} \\
& +\kappa \int_{0}^{L} d s u^{\prime \prime}(s)\left(y_{a}^{\prime \prime}(s)-\tilde{c}(s)\right)+f \int_{0}^{L} d s y_{a}^{\prime}(s) u^{\prime}(s) \\
& + \text { const. }
\end{aligned}
$$

Using the Euler-Lagrange equation (6), and the boundary conditions (5), one can easily show that the second line of the above equation vanishes. Therefore $\langle u(s)\rangle=$ $\left\langle u^{\prime}(s)\right\rangle=\left\langle u^{\prime \prime}(s)\right\rangle=0$, where $\langle\ldots\rangle$ denotes average with the weight $\exp (-\beta \mathcal{H}[u(s)]), \beta \equiv 1 / k_{B} T$.

We calculate $\left\langle u^{2}(s)\right\rangle$ and $\left\langle\left(u^{\prime}(s)\right)^{2}\right\rangle$ by decomposing $u(s)$ into Fourier harmonics which respect the prescribed boundary conditions: $u(s)=\sum_{n} a_{n} \sin (n \pi s / L), n \in \mathbb{N}$. This gives:

$$
\left\langle\left(u^{\prime}(s)\right)^{2}\right\rangle=\frac{2 k_{B} T}{L} \sum_{n=1}^{\infty} \frac{\cos ^{2}\left(\frac{n \pi}{L} s\right)}{\left[\kappa\left(\frac{n \pi}{L}\right)^{2}+f\right]}
$$

and

$$
\left\langle(u(s))^{2}\right\rangle=2 k_{B} T L \sum_{n=1}^{\infty} \frac{\sin ^{2}\left(\frac{n \pi}{L} s\right)}{\left[\kappa\left(\frac{n \pi}{L}\right)^{2}+f\right](n \pi)^{2}} .
$$

Note that the spontaneous curvature $\tilde{c}$ does not affect these thermal fluctuations. The decoupling of thermal and athermal curvature undulations and the results that we have obtained so far allow us to calculate various experimentally relevant observables such as force-extension curves and the average width of transverse fluctuations.

\section{FORCE-EXTENSION RELATIONSHIP}

The average projected length of the filament in the direction of the stretching force is given by

$$
\begin{aligned}
& \langle x(L)\rangle=L-\frac{1}{2} \int_{0}^{L} d s\left\langle t_{\perp}^{2}(s)\right\rangle \\
& =L-\frac{1}{2} \int_{0}^{L} d s\left(y_{a}^{\prime}(s)\right)^{2}-\frac{1}{2} \int_{0}^{L} d s\left\langle\left(u^{\prime}(s)\right)^{2}\right\rangle,
\end{aligned}
$$

where we have used the weakly bending approximation. There are four different force scales in the general problem,

$$
\begin{array}{rlrl}
f_{c r} & \equiv \frac{\kappa}{L_{p}^{2}}, & f_{c} \equiv \frac{\kappa}{c_{0}^{-2}} \\
f_{q} \equiv \frac{\kappa}{q^{-2}}, & f_{L} \equiv \frac{\kappa}{L^{2}}
\end{array}
$$

associated with the corresponding length scales: the persistence length $L_{p}=2 \kappa / k_{B} T$, the radius of spontaneous curvature $1 / c_{0}$, the wavelength of modulation of the spontaneous curvature $2 \pi / q$, and the contour length $L$. The force scale $f_{L}$ is related to finite-size effects in the sense that for $f \gg f_{L}$, or equivalently for $l_{f} \ll L$, the filament can be viewed as an effective freely jointed chain consisting of "pieces" each of contour length $l_{f}$ [23, 24]. In principle, the length scales $1 / c_{0}$ and $1 / q$ are independent (the former is the amplitude of the spontaneous curvature whereas the latter is the wavelength). However, the case where $c_{0} \gg q$ corresponds to very steep undulations and goes beyond the weakly bending approximation in the absence of a strong stretching force. The steepest undulations compatible with this approximation have $c_{0} \approx q$. On the other hand, we can be within the weakly bending approximation with $c_{0}>q$ if the stretching force is strong enough.

Let us first consider the case of constant spontaneous curvature $\tilde{c}=c_{0}$. In the limit where $f \gg f_{L}$, the forceextension relationship (15) acquires the simple form,

$$
\frac{\langle x(L)\rangle}{L}=1-\frac{1}{4}\left(\frac{L}{c_{0}^{-1}}\right)\left(\frac{f_{c}}{f}\right)^{3 / 2}-\frac{1}{2}\left(\frac{f_{c r}}{f}\right)^{1 / 2} .
$$

The first term in the right-hand side (rhs) of Eq. (17) is associated with athermal stretching whereas the second term is associated with the stretching of thermal fluctuations. The crossover force scale $\frac{1}{2}\left(L / c_{0}^{-1}\right)\left(f_{c}^{3 / 2} / f_{c r}^{1 / 2}\right) \sim$ $\kappa L_{p} L c_{0}^{4}$ determines the relative weight of the two contributions. In the strong stretching regime, the second term (which does not depend on the spontaneous curvature) becomes dominant in agreement with a similar result obtained recently in Ref. [19. If $L_{p} \approx L$ and the spontaneous curvature of the unstretched filament is small, $c_{0}^{-1} \gtrsim L$, the athermal contribution is almost negligible. It becomes significant, however, when the spontaneous curvature is large (the weakly bending approxi- 


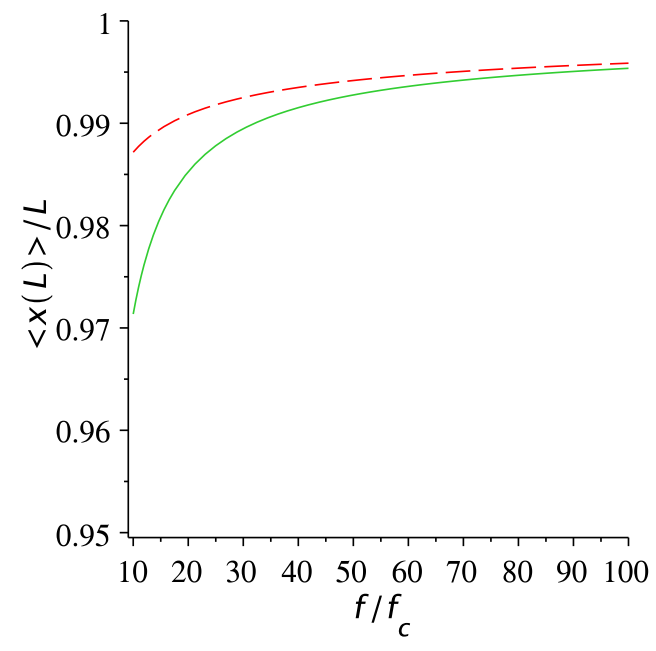

FIG. 2: Force-extension curve (solid line) for a filament with $L_{p} / L=1$ and $c_{0} L=12$. For comparison, we show the forceextension curve of the corresponding filament without spontaneous curvature (dashed line).

mation still applies to the stretched configuration of the filament). This is illustrated in Fig. 2

We now consider the case of a filament with spontaneous curvature which varies along its contour. If the local spontaneous curvature vanishes at the end-points, we can expand it in a sine series. In this case, the forceextension relationship takes the form,

$$
\begin{aligned}
& \frac{\langle x(L)\rangle}{L}=1-\frac{1}{2} \frac{\kappa^{2} \pi^{2}}{L^{2}} \sum_{n=1}^{\infty} \frac{c_{n}^{2} n^{2}}{\left[\kappa\left(\frac{n \pi}{L}\right)^{2}+f\right]^{2}} \\
& -\frac{1}{2} \frac{k_{B} T}{L} \sum_{n=1}^{\infty} \frac{1}{\left[\kappa\left(\frac{n \pi}{L}\right)^{2}+f\right]} .
\end{aligned}
$$

We see that as the stretching force increases, it successively irons out the larger length-scale (softer) modes of curvature (spontaneous or thermally excited) 25]. If the spontaneous curvature has only one mode and $f \gg f_{L}$, we obtain:

$$
\frac{\langle x(L)\rangle}{L}=1-\frac{1}{2} \frac{f_{q} f_{c}}{\left[f_{q}+f\right]^{2}}-\frac{1}{2}\left(\frac{f_{c r}}{f}\right)^{1 / 2} .
$$

This result holds irrespective of the value of the local spontaneous curvature at the end-points. As expected, when the spontaneous-curvature undulations have been

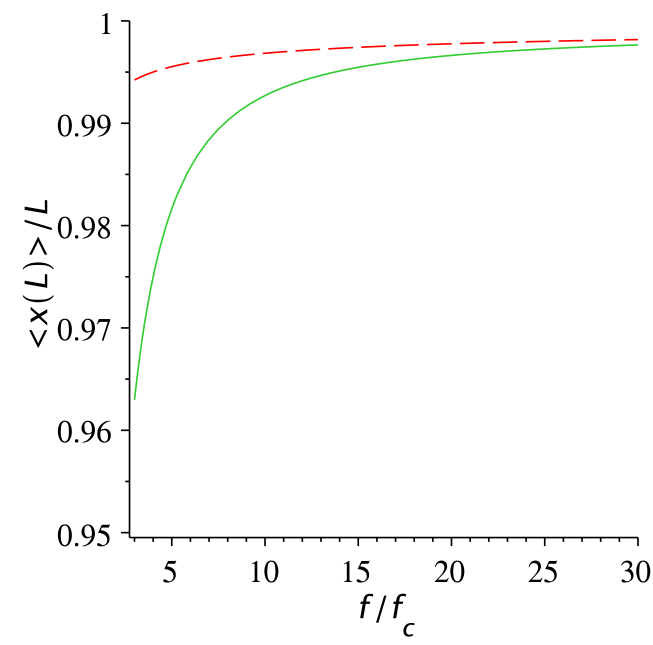

FIG. 3: Force-extension curve (solid line) for a filament with $L_{p} / L=0.05$ and $c_{0} L_{p}=q L_{p}=50$. For comparison, we show the force-extension curve of the corresponding filament without spontaneous curvature (dashed line).

ironed out by a strong force, the force-extension relationship is determined by the residual thermal fluctuations. The same is true when the persistence length is so small that thermal fluctuations wash out the spontaneous curvature. However, when $L_{p} \gg c_{0}^{-1} \approx q^{-1}$, the spontaneous curvature strongly affects the response to a stretching force. We point out that for $f_{L} \ll f_{c r} \ll f \lesssim f_{c} \approx f_{q}$ the response will be quite soft despite the nonlinear stretching of thermal undulations due to $f_{c r} \ll f$ as illustrated in Fig. 3. If the spatially variable spontaneous curvature contains many harmonic modes, our analysis still applies: Eqs. (9) and (15) yield a result similar to that of Eq. (18) but with the first sum in the rhs replaced by a more complicated term. For $f_{L} \gtrsim f$, the value of the local spontaneous curvature at the end-points only slightly affects the response as shown in Fig. 4.

We can gain insight into the effect of the spatially modulated spontaneous curvature on the strong stretching response by considering the effective freely jointed chain mentioned above. A two-dimensional freely jointed chain consisting of $N$ "pieces" of end-to-end distance $b$, has a force extension relationship,

$$
\frac{\langle x(L)\rangle}{N b}=1-\frac{k_{B} T}{2 f b},
$$

where $f \gg k_{B} T / b[23$. This equation can also be written 


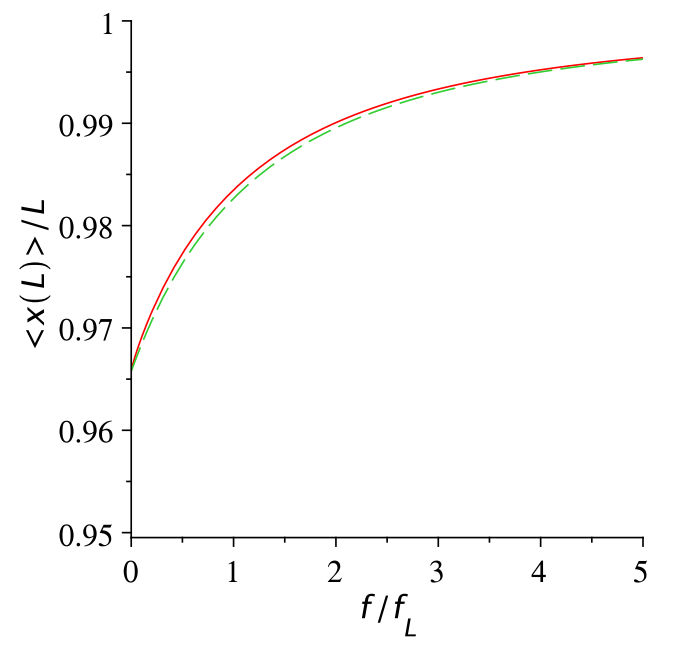

FIG. 4: Force-extension curve for a filament at $T=0$ with spontaneous curvature $\tilde{c}(s)=\frac{2.6}{L} \sin \left(\frac{10 \pi}{L} s\right)$ (solid line) and $\tilde{c}(s)=\frac{2.6}{L} \cos \left(\frac{10 \pi}{L} s\right)$ (dashed line).

as

$$
\frac{\langle x(L)\rangle}{L}=\frac{b}{2 l_{f}}-\frac{1}{2}\left(\frac{f_{c r}}{f}\right)^{1 / 2},
$$

where the chain is viewed as a succession of $N$ "pieces" of arc-length $2 l_{f}$. In the case of a WLC without spontaneous curvature, $b \equiv 2 l_{f}$ [23. In the case of a filament with spatially modulated spontaneous curvature, however, the "pieces" will be wavy with $b<2 l_{f}$. Plugging the result of Eq. (11) into the athermal part of Eq. (15), and assuming $q^{-1} \ll 2 l_{f}$, we obtain $b \equiv\left\langle x\left(2 l_{f}\right)\right\rangle \approx 2 l_{f}(1-$ $\left.f_{q} f_{c} / 2\left[f_{q}+f\right]^{2}\right)$, thus recovering Eq. 19 .

We note that in the case of spatially varying spontaneous curvature, the force-extension response for $f \gg f_{L}$ comes mainly from ironing out undulations in the bulk. Therefore we do not expect clamped boundary conditions of small compliance (e.g., with the direction of the clamped segments close to that of the pulling force), to significantly affect the response. On the other hand, as shown in Eq. (7), for a filament with constant spontaneous curvature and large persistence length in the strong stretching regime $\left(f \gg f_{L}\right)$, the response comes mainly from the boundary layers and the results will depend on the specific boundary conditions.

\section{TRANSVERSE FLUCTUATIONS}

Apart from the force-extension curve, the shape of transverse fluctuations can be a useful diagnostic tool for the elasticity of a semiflexible filament [26]. For a fluctuating filament with spontaneous curvature $\tilde{c}(s)=$ $c_{0} \sin (q s)(q=n \pi / L)$, the average width of transverse fluctuations along the polymer contour is given by

$$
\begin{aligned}
& \frac{1}{L} \int_{0}^{L} d s\left\langle(y(s))^{2}\right\rangle=\frac{\kappa f_{c}}{\left(f+f_{q}\right)^{2}} \\
& +L k_{B} T \frac{(\tilde{f})^{3 / 2}-3 \operatorname{coth}(\sqrt{\tilde{f}}) \tilde{f}+3 \sqrt{\tilde{f}}}{6 f_{L}(\tilde{f})^{5 / 2}}
\end{aligned}
$$

where the non-dimensional shorthand $\tilde{f} \equiv f / f_{L}$ is used. The first (athermal) term is related to the spontaneous curvature and the second term comes from the thermal fuctuations. For $f \gg f_{L}$, the latter can be approximated by $\frac{1}{6} L k_{B} T / f$.

The profile of transverse fluctuations has the general form:

$$
\begin{aligned}
& \frac{\left\langle(y(s))^{2}\right\rangle}{L}=\frac{c_{0}^{2} L^{2}}{\left(f / f_{L}\right)^{2}} F\left(f / f_{L, q}, s / L\right) \\
& +\frac{L}{L_{p}} G\left(f / f_{L}, s / L\right),
\end{aligned}
$$

where $F$ involves $f_{L}$ or $f_{q}$ depending on whether the spontaneous curvature is constant or oscillating. In the absence of spontaneous curvature, the profile of transverse fluctuations is determined by the function $G\left(f / f_{L}, s / L\right) \propto\left\langle(u(s))^{2}\right\rangle$ given in Eq. 14) which is bell-shaped and its height decreases rather quickly with increasing force. This also applies when the stretching force is so strong that it has ironed out the undulations due to the spontaneous curvature. Interestingly, this behavior persists even for stretching forces such that $f_{L} \ll f_{c r} \ll f \ll f_{c} \sim f_{q}$ where, as we have shown, the force-extension relationship is strongly affected by the spontaneous-curvature contribution. The behavior becomes qualitatively different in the case of rather stiff filaments $\left(L_{p} \gg L\right)$ for $f \ll f_{q} \sim f_{c}$ and $f / f_{q} \gg L / L_{p}$. The athermal term becomes dominant and it decreases very slowly with increasing force. In addition, the profile is essentially flat, characterized by the constant amplitude of the sinusoidal undulations. This type of behavior is expected also in the case where $f_{c} \gg f_{q}$ and $f \ll f_{q}$ such that $f_{c} / f_{q}^{2} \gg L /\left(L_{p} f\right)$. In order to get $f_{c} \gg f_{q}$, we need a filament with very steep undulations of the spontaneous curvature $\left(c_{0} \gg q\right)$. This would take us out of validity of the weakly bending approximation. Although we cannot make quantitative predictions for this case, we expect the main qualitative features of the predicted behavior to persist. 


\section{CONCLUSIONS AND OUTLOOK}

In the present work, we considered a straightforward extension of the WLC model which incorporates spontaneous curvature to study the response of a twodimensional weakly bending filament to a pulling force applied at its ends. The response of a filament with intermediate flexibility $\left(L / L_{p} \approx 1\right)$ and constant spontaneous curvature is affected appreciably only for large initial curvature, where the effective stretching modulus of the chain softens. If the spontaneous curvature is inhomogeneous, characterized by undulations of a characteristic wavelength, we find a range of forces where these undulations soften the response despite the nonlinear flattening out of thermal fluctuations. The profile of transverse fluctuations is mostly dominated by the bell-shaped thermal contribution unless the spontaneous curvature undulations are very steep.

Although our quantitative results are based on the twodimensional weakly bending assumptions, the qualitative features are expected to hold more generally as they are related to the length and force scales of the problem. As we have shown in Sec. $\mathrm{V}$, the softening of the stretching response for $f_{L} \ll f_{c r} \ll f \ll f_{c} \approx f_{q}$ can be attributed to the fact that the "pieces" of the effective freely jointed chain have an end-to-end distance smaller than the corresponding contour length because of their spontaneous curvature. One might expect a similar hierarchy of length and force scales to persist in the case of a three-dimensional stretched helical filament with the pitch length and the radius playing roles analogous to $q^{-1}$ and $c_{0}^{-1}$, respectively, thus yielding a similar softening. Checking the validity of this expectation is an interesting direction for future work.

\section{ACKNOWLEDGMENTS}

P.B. gratefully acknowledges support from EPSRC via the University of Cambridge TCM Programme Grant.

[1] J. Howard, Mechanics of Motor Proteins and the Cytoskeleton, (Sinauer Associates, Sunderland, MA, 2001).
[2] P. Nelson, Biological Physics; Energy, Information, Life, (W. H. Freeman \& Co., New York, NY, 2004).

[3] O. Kratky and G. Porod, Recl. Trav. Chim. Pays-Bas 68, 1106 (1949).

[4] N. Saitô, K. Takahashi and Y. Yunoki, J. Phys. Soc. Jpn. 22, 219 (1967).

[5] J. R. Blundell and E. M. Terentjev, Macromolecules 42, 5388 (2009).

[6] C. Bustamante, S. B. Smith, J. Liphardt, and D. Smith, Curr. Opin. Struct. Biol. 10, 279 (2000), and references therein.

[7] B. Ghosh and A. Sain, Phys. Rev. Lett. 101, 178101, (2008).

[8] H. P. Erickson, Proc. Natl. Acad. Sci. U.S.A. 106, 9238, (2009).

[9] A. Dajkovic, G. Lan, S. X. Sun, D. Wirtz, and J. Lutkenhaus, Curr. Biol. 18, 235, (2008).

[10] I. Horger, E. Velasco, J. Mingorance, G. Rivas, P. Tarazona, and M. Velez, Phys. Rev. E 77, 011902 (2008).

[11] T. Ohyama (Ed.), DNA Conformation and Transcription, (Springer, New York, NY, 2005).

[12] W. Han, M. Dlakic, Y.-J. Zhu, S. M. Lindsay, and R. E. Harrington, Proc. Natl. Acad. U.S.A. 94, 10565 (1997)

[13] J. Moukhtar, E. Fontaine, C. Faivre-Moskalenko, and A. Arneodo, Phys. Rev. Lett. 98, 178101 (2007).

[14] S. Panyukov and Y. Rabin, Phys. Rev. Lett. 85, 2404, (2000).

[15] A. Craig and E. M. Terentjev, Macromolecules 39, 4557, (2006).

[16] D. A. Kessler and Y. Rabin, J. Chem. Phys. 118, 897, (2003).

[17] D. A. Kessler and Y. Rabin, Phys. Rev. Lett. 90, 024301, (2003).

[18] H. Wada and R. R. Netz, Europhys. Lett. 77, 68001, (2007).

[19] Z. Zhou, Phys. Rev. E 76, 061913 (2007).

[20] B. Maier, U. Seifert, and J. O. Rädler, Europhys. Lett. 60, 622, (2002).

[21] A. Prasad, Y. Hori and J. Kondev, Phys. Rev. E 72, 041918 (2005).

[22] Z. Zhou and B. Joós, Phys. Rev. E 80, 061911 (2009).

[23] J. Kierfeld, O. Niamploy, V. Sa-yakanit, and R. Lipowsky, Eur. Phys. J. E 14, 17, (2004).

[24] P. G. de Gennes, in Polymer Liquid Crystals, edited by A. Ciferri, W. R. Kringbaum and R. B. Meyer (Academic Press, New York, 1982) Chapter 6.

[25] A. Kabla and L. Mahadevan, J. R. Soc., Interface 4, 99, (2007).

[26] P. K. Purohit, M. E. Arsenault, Y. Goldman and H. H. Bau, Int. J. Non Linear Mech. 43, 1056 (2008). 